\title{
BENEFÍCIOS DO PARTO NORMAL PARA A QUALIDADE DE VIDA DO BINÔMIO MÃE- FILHO
}

\author{
BENEFITS OF NATURAL CHILDBIRTH FOR THE QUALITY OF LIFE OF THE MOTHER-CHILD \\ BINOMIAL
}

\author{
Rebeca Cardoso Gazineu ${ }^{1}$ \\ Karla Rocha de Almeida Amorim² \\ Camila Torres da Paz ${ }^{3}$ \\ Rita de Cássia Calfa Vieira Gramacho ${ }^{4}$
}

\begin{abstract}
Este estudo teve como objetivo analisar de que forma o parto normal contribui para uma melhor qualidade de vida do binômio mãe-bebê. Trata-se de uma revisão de literatura integrativa, cujos artigos foram selecionados na Biblioteca Virtual de Saúde. Dos 82 encontrados, 17 abordavam o tema proposto e foram selecionadas para compor este estudo. Constatou-se que os anos que apresentaram maior número de artigos publicados foram 2014 e 2015, com seis e cinco publicações, respectivamente, correspondendo a $64.7 \%$ de publicações incluídas no estudo. O ano de 2016 aparece com quatro publicações e 2017 com duas. Após a análise dos dados, surgiram três categorias: a. O ciclo gravídico e a escolha da via de parto; b. Fatores que interferem na escolha do tipo de parto; e c. Vantagens e desvantagens do parto normal. Os estudos revelaram que o ciclo gravídico é uma fase marcante na vida da mulher, que deve tomar decisões significativas que contribuem para uma melhor qualidade de vida do binômio mãe-bebê. Foi possível entender que muitas mulheres optam pela cesariana em detrimento do parto normal, e que este fenômeno pode ser explicado por diversos fatores, como o déficit de informações durante a assistência. Conclui-se que há uma necessidade de incentivo ao parto normal, já que suas vantagens superam suas desvantagens. Por outra parte, os profissionais de saúde devem promover a educação em saúde, incentivando o parto natural e ressaltando os seus benefícios.
\end{abstract}

Palavras-chave: Parto. Parto Normal. Parto Humanizado.

This study aimed to review how normal delivery contributes to a better life quality of the mother-baby binomial. An integrative literature review of scientific articles selected in electronic databases, through the Virtual Health Library was carried out. Eighty two articles were found, of which 17 approached the proposed theme and were selected to compose this study. Most of the research papers were published in 2014 and 2015, with six and five publications respectively, corresponding to $64.7 \%$ of the publications included in the study. After analyzing the data, three categories emerged: the pregnancy cycle and the choice of the delivery route; factors that interfere in the choice of delivery type; and advantages and disadvantages of normal delivery. Studies have shown that the pregnancy cycle is a remarkable phase in a woman's life, in which she has to make significant decisions that contribute to a better quality of life of the mother-baby binomial. It was possible to understand that many women opt for cesarean section at the expense of normal delivery, and that this phenomenon can be explained by several factors, such as the deficit of information during antenatal care. It is concluded that there is a need to encourage normal childbirth, since its advantages outweigh its disadvantages in terms of health benefits.

Keywords: Childbirth. Normal Birth. Humanized Birth.

\footnotetext{
1Enfermeira; Pós Graduanda em Enfermagem Obstétrica (EBMSP); Salvador; Bahia; E-mail: becagazineu@gmail.com; Currículo Lattes: http://lattes.cnpq.br/3833982873309484.

²Enfermeira; Pós Graduanda em Enfermagem Obstétrica (EBMSP); Salvador; Bahia; E-mail: karla_rochaa@hotmail.com; Currículo Lattes: http://lattes.cnpq.br/2266121011734536.

${ }^{3}$ Enfermeira Obstetra ; Orientadora da pesquisa; Mestra em Desenvolvimento Regional e Meio Ambiente - Faculdade Maria Milza (FAMAM); Docente da Faculdade Maria Milza (FAMAM); Cruz das Almas; Bahia; E-mail: camilatorrespaz@gmail.com; Currículo Lattes: http://lattes.cnpq.br/1880862855767805.

${ }^{4}$ Enfermeira Obstetra; Coordenadora do Curso de Pós Graduação em Enfermagem Obstétrica da Escola Bahiana de Medicina e Saúde Pública (EBMSP); Diretora Geral da Maternidade Tsylla Balbino; Salvador; Bahia; E-mail:ritacalfa@hotmail.com; Currículo Lattes:

http://lattes.cnpq.br/2581318156352565.
} 


\section{INTRODUÇÃO}

A gestação é um período fisiológico que envolve transformações físicas e emocionais para a mulher (GUEDES et al., 2016), podendo ser caracterizada como um momento de adequação, que se deve à preparação do corpo para gerar e nutrir o feto, dando muitas vezes continuidade a costumes e crenças que se perpetuam durante gerações, como a de que o relacionamento sexual durante a gestação pode afetar a criança. Dessa forma, a gestante passa a sofrer influência da sociedade, sendo obrigada a adequar-se ao ideal imposto, interligando o passado e o presente (CAMPOS; ALMEIDA; SANTOS, 2014). Este fato, atrelado ao déficit de informação oferecida a essas mulheres, gera incertezas, dúvidas e insegurança quanto ao momento do parto (SILVA; PRATES; CAMPELO, 2014).

O ciclo gravídico e o processo de parturição sofreram modificações com o passar do tempo, visto que, apesar de ser um fenômeno natural, acabou tornando-se um evento medicalizado (SANTOS et al., 2016). O parto, que antes era realizado em ambiente domiciliar, com auxílio de parteiras e sem ações intervencionistas, tornou-se um ato comercial (CAMPOS; ALMEIDA; SANTOS, 2014).

Segundo Melo, Davim e Silva (2015), o aumento do número de cesáreas, atrelado às consequências negativas deste procedimento, fez com que a Organização Mundial de Saúde (OMS) estabelecesse uma taxa de cesariana padrão entre 10 e 15\% do total de partos, o que levou o Ministério da Saúde (MS) a estimular o parto normal. Os Indicadores e Dados Básicos (IDB) para a saúde do Brasil fornecem, em seus índices de cobertura, a proporção de partos cesáreos, cujos valores alcançaram o patamar de 52,34\%, no ano de 2010 (VELHO; SANTOS; COLLAÇO, 2014, p. 283). A escolha por essa via de parto, muitas vezes, está relacionada ao medo, à conveniência e à desinformação (NASCIMENTO et al., 2015).

O MS brasileiro prioriza a assistência humanizada em todo o pré-parto, parto e pós-parto (PPP), assistindo a mãe e criança por completo. Além disso, estabelece que para que se tenha uma assistência humanizada, o ideal é que haja a menor intervenção possível no período de parturição, resgatando a valorização da fisiologia do parto, priorizando a segurança do binômio mãe-filho, de forma que o processo seja saudável para ambos (VELHO; SANTOS; COLLAÇO, 2014).

Para que isso se concretize, faz-se necessário que essas mulheres tenham conhecimento de seus direitos, e que participem no ciclo gravídico tomando decisões com autonomia, segurança e controle, de forma a garantir a singularidade do nascimento. A mulher, quando embasada por essas informações, contribui com a melhoria na assistência e participa mais das decisões, podendo desfrutar de uma gestação mais segura, tranquila e que, consequentemente, promoverá a formação do vínculo mãe-filho e facilitará a aceitação da sua gravidez (VELHO; SANTOS; COLLAÇO, 2014).

Diante deste contexto, este estudo visa indagar os fatores que têm levado as mulheres à escolha da prática medicalizada do parto e ao abandono do parto normal.

Acredita-se que o abandono do parto normal possa estar relacionado ao receio da dor durante o trabalho de parto, à praticidade da cesariana (trabalho de parto curto), e à utilização de analgésicos durante o processo.

Sendo assim, este estudo tem como objetivo geral revisar de que forma o parto normal contribui para uma melhor qualidade de vida do binômio mãe-bebê. Logo, como objetivos específicos tem-se: Identificar a importância do parto normal para o desenvolvimento do bebê e relacionar os benefícios que o parto normal tem para a mulher.

Portanto, este estudo se justifica por analisar de que forma o parto normal está sendo visto e entendido pela sociedade, principalmente pelas mulheres, além de esclarecer a importância deste método de parturição para a saúde da mãe e do bebê, encorajando a mulher a ser a personagem principal desse processo. 


\section{METODOLOGIA}

Este estudo foi elaborado a partir de uma revisão integrativa da literatura, que consiste em uma reavaliação de resultados obtidos em estudos anteriores a respeito do tema em questão (SOARES et al., 2014).

O levantamento dos artigos ocorreu em fevereiro de 2018, na Base de Dados da Biblioteca Virtual em Saúde (BVS). Os descritores empregados foram: parto, parto normal, parto humanizado.

Os critérios de inclusão estabelecidos foram: artigos completos em língua portuguesa, disponíveis eletronicamente, publicados entre 2014 e 2018 em periódicos da área de interesse e em formato de artigo. Após serem atendidos os critérios, foi totalizado um universo de 82 artigos, sendo 29 na base de dados LILACS, 44 na base de dados BDENF e 09 na base de dados MEDLINE, como pode ser visto no Quadro 1.

Quadro 1. Resultados das buscas nas bases de dados eletrônicas e seleção dos títulos relacionados ao assunto em questão.

\begin{tabular}{|c|c|c|}
\hline Bases de Dados & Total & Aceitos \\
\hline BDENF & 44 & 8 \\
\hline LILACS & 29 & 7 \\
\hline MEDLINE & 9 & 2 \\
\hline
\end{tabular}

Fonte: Dados de Pesquisa, 2018.

Foram encontrados 82 artigos, dos quais 13 apresentaram-se repetidos em mais de uma base. Os artigos que se repetiram em duas bases de dados foram agregados na base de dados que apareceu primeiro no momento da pesquisa. Assim, das 82 publicações elencadas, 17 abordavam o tema proposto e foram selecionadas para compor este estudo (Quadro 2).

Quadro 2. Descrição dos estudos incluídos na revisão integrativa, segundo título, autor (es), base de dados e ano de publicação.

\begin{tabular}{|l|l|l|l|c|}
\hline$N^{\circ}$ & \multicolumn{1}{|c|}{ Título } & $\begin{array}{c}\text { Base de } \\
\text { Dados }\end{array}$ & \multicolumn{1}{|c|}{ Autores } & $\begin{array}{c}\text { Ano de } \\
\text { Publicação }\end{array}$ \\
\hline 1 & $\begin{array}{l}\text { Fatores que influenciam a escolha do tipo de } \\
\text { parto na percepção das puérperas }\end{array}$ & LILACS & FEITOSA, R. M. M et al. & 2017 \\
\hline 2 & $\begin{array}{l}\text { Parto normal assistido por enfermeira: } \\
\text { experiência e satisfação de puérperas }\end{array}$ & BDENF & FREIRE, H. S. de S. et al. & 2017 \\
\hline 3 & $\begin{array}{l}\text { Conhecimentos de gestantes quanto aos } \\
\text { benefícios do parto normal na consulta prénatal }\end{array}$ & BDENF & GUEDES, G. W. et al. & 2016 \\
\hline 4 & $\begin{array}{l}\text { Preparo e percepções de gestantes sobre as } \\
\text { vias de parto }\end{array}$ & BDENF & SANTOS C. L dos. et al. & 2016 \\
\hline 5 & $\begin{array}{l}\text { Nascimentos no Brasil: associação do tipo de } \\
\text { parto com variáveis temporais e } \\
\text { sociodemográficas }\end{array}$ & LILACS & $\begin{array}{l}\text { RATTNER, D.; MOURA, } \\
\text { E. C de. }\end{array}$ & 2016 \\
\hline 6 & $\begin{array}{l}\text { Fatores que influenciam na indicação da via de } \\
\text { parto }\end{array}$ & LILACS & PINHEIRO, T. M. et al. & 2016 \\
\hline 7 & $\begin{array}{l}\text { Vantagens e desvantagens do parto normal e } \\
\text { cesariano: opinião de puérperas }\end{array}$ & BDENF & $\begin{array}{l}\text { MELO, J. K. F de.; DAVIM, } \\
\text { R. M. B. }\end{array}$ & 2015 \\
\hline 8 & $\begin{array}{l}\text { Percepção de puérperas sobre a assistência à } \\
\text { saúde em um centro de parto normal }\end{array}$ & BDENF & RIBEIRO, J. F. et al. & 2015 \\
\hline 9 & $\begin{array}{l}\text { "No final compensa ver o rostinho dele": } \\
\text { vivências de mulheres -primíparas no parto } \\
\text { normal. }\end{array}$ & MEDLINE & SCARTON, J. et al. & 2015 \\
\hline 10 & $\begin{array}{l}\text { Parto natural X parto cirúrgico: percepções de } \\
\text { mulheres que vivenciaram os dois momentos }\end{array}$ & BDENF & $\begin{array}{l}\text { CARNEIRO, L. M. de A. et } \\
\text { al. }\end{array}$ & 2015 \\
\hline
\end{tabular}




\begin{tabular}{|c|l|c|l|c|}
\hline 11 & $\begin{array}{l}\text { Escolha do tipo de parto: fatores relatados por } \\
\text { puérperas }\end{array}$ & LILACS & $\begin{array}{l}\text { NASCIMENTO, R. R. P. } \\
\text { do. et al. }\end{array}$ & 2015 \\
\hline 12 & $\begin{array}{l}\text { A cultura interferindo no desejo sobre o tipo de } \\
\text { parto }\end{array}$ & LILACS & PIMENTA, L. F. et al. & 2014 \\
\hline 13 & $\begin{array}{l}\text { O comportamento expresso pela parturiente } \\
\text { durante o trabalho de parto: reflexos da } \\
\text { assistência do pré-natal }\end{array}$ & LILACS & MELO, K. de L. et al. & 2014 \\
\hline 14 & $\begin{array}{l}\text { A assistência humanizada no trabalho de parto: } \\
\text { percepção das adolescentes }\end{array}$ & LILACS & VARGAS, P. B. et al. & 2014 \\
\hline 15 & $\begin{array}{l}\text { Crenças, mitos e tabus de gestantes acerca do } \\
\text { parto normal }\end{array}$ & BDENF & $\begin{array}{l}\text { CAMPOS, A. S.; } \\
\text { ALMEIDA, A. C. } \text {. H de.; } \\
\text { SANTOS, R. P. dos. }\end{array}$ & 2014 \\
\hline 16 & $\begin{array}{l}\text { Parto normal e cesárea: representações sociais } \\
\text { de mulheres que os vivenciaram. }\end{array}$ & MEDLINE & $\begin{array}{l}\text { VELHO, M. B.; dos } \\
\text { SANTOS, E. K. A.; } \\
\text { COLLAÇO, V. S }\end{array}$ & 2014 \\
\hline 17 & $\begin{array}{l}\text { Parto normal ou cesariana? Fatores que } \\
\text { influenciam na escolha da gestante }\end{array}$ & BDENF & $\begin{array}{l}\text { SILVA, S. P.; PRATES, R. } \\
\text { de C. G; CAMPELO, B. Q. } \\
\text { A }\end{array}$ & 2014 \\
\hline
\end{tabular}

Fonte: Dados de Pesquisa, 2018.

Quanto ao período de publicação, constatou-se que os anos que apresentaram maior número de artigos publicados foram 2014 e 2015, com seis e cinco publicações, respectivamente, correspondendo a $64.7 \%$ de publicações incluídas no estudo. O ano de 2016 aparece com quatro publicações e 2017 com duas.

Em relação ao delineamento de pesquisa, identificou-se que das 17 publicações, 15 utilizaram abordagens qualitativas (88.2\%), 01 quantitativa (5.8\%) e 01 delineamento misto, composto por um estudo de série temporal descritivo (5.8\%). Portanto, a abordagem metodológica dos estudos aponta uma forte tendência para realização de pesquisas qualitativas sobre a temática.

Com relação à autoria, os 17 trabalhos analisados foram publicados por 83 autores, sendo 71 do sexo feminino e 12 do sexo masculino. O diferencial do número de autores em relação ao número de trabalhos publicados ocorreu devido à multiautoria das publicações em periódicos, com notável predomínio do sexo feminino.

\section{RESULTADOS E DISCUSSÕES}

Após a análise dos dados a partir de uma leitura aprofundada dos artigos selecionados, surgiram três categorias: 1) O ciclo gravídico e a escolha da via de parto; 2) Fatores que interferem na escolha do tipo de parto e; 3) Vantagens e desvantagens do parto normal.

\section{O CICLO GRAVÍDICOEAESCOLHADAVIADE PARTO}

O ciclo gravídico puerperal é uma experiência significativa na vida da mulher e de seus familiares; logo, pode vir associada a sentimentos divergentes, chamados por Velho, Santos e Collaço (2014, p 285) de "ambivalência de sentimentos" ou momento de sensações mistas (boas ou ruins), dependendo de como sejam vividas e percebidas por cada mulher (MELO et al., 2014; FREIRE et al., 2017). Essa ambivalência, quando voltada para o parto normal, é ainda definida como as dificuldades enfrentadas no trabalho de parto pela mulher quando comparadas às facilidades de sua recuperação (VELHO; SANTOS; COLLAÇO, 2014).

Durante a gestação, a mulher possui grande influência na decisão de qual tipo de parto será realizado. Apesar de ser um direito, a gestante necessita receber informações fidedignas a respeito das vias de parto, com enfoque nas vantagens e desvantagens de cada tipo. Nesse sentido, o diálogo entre o profissional de saúde e a mulher permite a negociação e a troca de saberes, a partir do esclarecimento de 
dúvidas, mitos e medos, para que assim a gestante possa tomar decisões de forma segura e com autonomia (SILVA; PRATES; CAMPELO, 2014; PINHEIRO et al., 2016).

Para Pinheiro et al. (2016), a escolha da via de parto, seja ela vaginal ou cesariana, possui justificativas de relevância, tal como o receio da dor. Além disso, Santos et al. (2016) complementam que existe a expectativa atrelada ao conhecimento que essas mulheres possuem sobre o assunto e às informações que são passadas pelos profissionais que as atendem durante todo o período, visto que o processo de preparação da futura mãe para o parto começa desde a primeira consulta de pré-natal. Não obstante, Silva, Prates e Campelo (2014) referem que a liberdade de escolha assegurada à mulher, na maioria das vezes, se torna ilusória, uma vez que as mulheres são influenciadas pelas informações fornecidas pelos profissionais de saúde que acompanham o ciclo gravídico através do pré-natal.

É evidente que a realização de atividades educativas nos serviços de saúde durante o pré-natal, baseadas na educação em saúde, são imprescindíveis para a construção do aprendizado que permitirá à mulher escolher de forma autônoma a via de parto que mais deseja (SANTOS et al., 2016).

Vale destacar que os tipos de parto disponíveis são a cesariana e o parto normal. Todavia, a crescente porcentagem de cesarianas é um fator que preocupa os gestores das unidades de saúde, especialmente ao se ponderar os riscos desnecessários a que essas parturientes e seus bebês estão sujeitos quando se ultrapassam as reais condições que justificam uma cesariana, ou seja, quando o parto cirúrgico é realizado sem fundamento (NASCIMENTO et al., 2015).

Segundo Rattner e Moura (2016), a Rede Cegonha, estratégia implantada pelo MS para implementar uma rede de cuidados à mulher e à criança, alerta quanto à mudança no modelo de atenção, fazendo-se necessária a implantação de um verdadeiro monitoramento dos procedimentos cirúrgicos, para que diminuam as taxas nos grupos de menor risco. Os incentivos à realização de partos normais e naturais são necessários quando levados em consideração os benefícios para mãe e o bebê, visando à diminuição do número de cesarianas desnecessárias.

Ainda de acordo com os autores supracitados, estes incentivos devem começar na formação dos profissionais, sejam eles enfermeiros ou médicos, tendo como objetivo, principalmente, a humanização do atendimento e a promoção da autonomia e empoderamento da mulher, para a conscientização dessas mães em relação a benefícios como a rápida recuperação, redução das dores no pós-parto, favorecimento do vínculo entre mãe e bebê através do contato e aleitamento materno na primeira hora, e consequente retorno mais rápido à rotina.

Assim sendo, nota-se que a escolha pela via de parto deve ser embasada em informações verdadeiras, fundamentadas em um conhecimento científico crítico, a partir de um pré-natal de qualidade, para que a mulher se sinta segura para optar pelo melhor tipo de parto para ela.

\section{FATORES QUE INTERFEREM NAESCOLHADO TIPO DE PARTO}

A escolha pelo tipo de parto tem relação com diversos fatores, tais como o déficit de informações com que conta a mulher a respeito do pré-parto, parto e puerpério; os processos de parturição; a dor e as possíveis lacerações vaginais, que levam, frequentemente, a uma cesariana eletiva.

No estudo 1 ficou evidenciado que dentre os fatores que interferem na escolha da mulher pelo tipo de parto estão incluídos os riscos, as vantagens e desvantagens, e possíveis consequências, sejam elas relacionadas ou não à condição de parto Estes aspectos só podem ser abordados se houver uma boa qualidade de assistência geral e obstétrica e uma visão integral dessa gestante e do bebê ao longo do pré natal. Essa qualidade na assistência envolve o direito de livre escolha da gestante, que é orientada sobre todos os processos envolvidos, como preconiza o Programa de Humanização no Pré-natal e Nascimento.

Em convergência, Campos, Almeida e Santos (2014) constataram que a cultura e os princípios de amigos e familiares interferem expressivamente na decisão do tipo de parto. Este pensamento condiz com o estudo 6, que assegura que os costumes e valores transmitidos de geração em geração, dependendo da forma como foram passados, podem vir a influenciar a tomada de decisão. Também, o estudo 16 conclui que tais aspectos estão atrelados a crenças e mitos, divulgados nas redes sociais, que transmitem todo tipo de informações. 
Outro fator preponderante que interfere no critério de escolha é o déficit na comunicação entre a equipe de saúde e a gestante e seus familiares durante a preparação para o parto e puerpério. Esta afirmação é sustentada por Freire et al. (2017, p. 2364) ao afirmar que "a falta de conhecimento proveniente da ausência de orientação ou da orientação insuficiente durante o pré-natal dificulta o enfretamento da parturição pela mulher por temer o desconhecido".

Sabe-se que a consulta do profissional que atende no pré-natal tem como objetivo primordial a informação, o aconselhamento, a educação e contribuição para a prevenção de possíveis complicações para a mãe e o bebê, contribuindo para a promoção da saúde, pautada em uma assistência integral e humanizada, voltada para as necessidades individuais de cada mulher (GUEDES et al., 2016). Desta maneira, uma assistência humanizada, de acordo com Vargas et al. (2013), propicia o estabelecimento do vínculo mãe-filho já nos primeiros minutos de vida da criança.

O profissional que assiste a mãe tem um papel relevante como educador em saúde, pois transmite segurança e a auxilia na tomada de decisão consciente da sua via de parto (SILVA; PRATES; CAMPELO, 2014). Não obstante, ainda se percebe falta de precisão nas informações dadas e baixo nível de conhecimento das gestantes, que resultam na falta de confiança para fazer a escolha mais apropriada (FEITOSAet al., 2017).

Ao participar de forma ativa, as mulheres podem manifestar seus saberes, embora empíricos, ao mesmo tempo em que os profissionais de saúde compartilham seus conhecimentos científicos. Nesse contexto, constrói-se um somatório de aprendizados, que podem contribuir para que a mulher e sua família vivenciem plenamente o período gravídico-puerperal (SANTOS et al., 2016, p 191).

Assim, notou-se que a comunicação efetiva, através de um olhar integral e humanizado é essencial para a existência de uma gestação tranquila e satisfatória, do ponto de vista de conhecimento, evitando possíveis transtornos devido à falta de informação, proporcionando à mulher uma participação mais ativa no seu ciclo gravídico.

\section{VANTAGENS E DESVANTAGENS DO PARTO NORMAL}

Assim como todo e qualquer procedimento, o parto normal possui vantagens e desvantagens tanto para as mães quanto para os bebês, e precisam ser explicadas minuciosamente durante a assistência de saúde.

As experiências da gestação e do nascimento, quando bem acompanhadas e esclarecidas, são capazes de influenciar positivamente na autoestima feminina, além de favorecer a autonomia e empoderamento da mulher (FREIRE et al., 2017). Na opinião de Scarton et al. (2015), apesar de o nascimento do bebê estar atrelado à intensas expectativas, não se pode ignorar o fato de que também envolve ansiedade e medo pelo desconhecido. Em concordância, o estudo 13 refere que o medo do desconhecido, da dor intensa ou de estar desacompanhada, tem como consequência a preocupação da segurança e bem-estar da própria mulher e do seu bebê.

"A assistência de qualidade à mulher em trabalho de parto é um direito fundamental e representa um passo indispensável para garantir que ela possa exercer a maternidade com segurança e bem-estar" (RIBEIRO et al., 2015, p.523).

Nesse viés, percebe-se a importância da informação referente às vantagens e desvantagens de cada tipo de parto para a parturiente, principalmente com incentivo para o parto normal, explicando a essência da fisiologia do processo, fazendo com que entenda suas possibilidades e estimulando-a a ser protagonista deste momento ímpar e sublime, favorecendo então uma parturição mais leve e prazerosa (GUEDES et al., 2016).

O parto normal é incomparavelmente mais saudável, com a grande vantagem de tornar a mulher a protagonista dessa etapa. O corpo feminino foi preparado fisiologicamente para o processo de parturição. Por isso, é capaz de enfrentar este momento sem a necessidade de tecnologias interventistas. Além disso, os benefícios que esta via de parto proporciona para a mulher e o bebê são 
imensuráveis (NASCIMENTO et al., 2015.; GUEDES et al., 2016).

Estudos realizados por Carneiro et al. (2015) evidenciaram que o benefício mais relatado por puérperas em relação ao parto normal é o fato de se ter uma recuperação mais rápida no pós parto. Pimenta et al. (2014) e Melo, Davim e Silva (2015) também confirmam esses resultados. Segundo Velho, Santos e Collaço (2014, p. 285-286), a recuperação da mulher é mais acelerada e melhor tolerada, visto que garante "independência para caminhar, realizar cuidados de higiene pessoal e atividades domésticas, cuidar do bebê, além de uma recuperação mais rápida do seu próprio corpo".

Outros benefícios também são percebidos no processo natural de parturição, tais como aumento do contato do binômio mãe-bebê, que faz com que a criança fique aquecida e que perca menos energia; contribuição na estabilização cardíaca e respiratória do bebê, evitando o risco de adquirir taquipneia transitória e síndrome respiratória, além de diminuir o estresse (CARNEIRO et al., 2015), o que fortalece o vínculo no binômio e auxilia para uma melhor qualidade de vida de ambos. Por outra parte, Guedes et al. (2016) apontam que outra consequência positiva do parto natural é o fato da mulher e o bebê correrem menos risco de adquirir infecções, devido aos mecanismos fisiológicos propiciados pelo processo de parturição.

Em relação às desvantagens do parto vaginal, destaca-se a sensação de dor na maioria dos artigos encontrados. Conforme Freire et al. (2017), a dor pode ser elevada de acordo com determinados sentimentos, como medo, ansiedade, solidão, entre outros, mas desaparecem com o pós-parto. A dor do parto é caracterizada por Pimenta et al. (2014, p. 991) como uma "grande medida iatrogênica", ou seja, danos que poderiam ser evitados, demonstrada através de uma assistência que introduz medidas desnecessárias, tal como o uso demasiado de ocitocina artificial, a manobra de Kristeller*, a

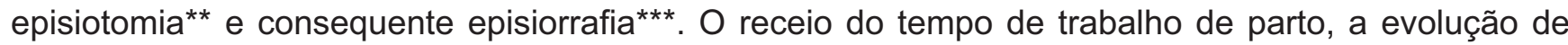
complicações também são desvantagens que podem ocorrer durante a parturição (MELO; DAVIM, 2015).

Muitas mulheres ainda sentem medo de parirem por via vaginal, sobretudo por temerem as consequências que podem advir desta via de parto como, por exemplo, o desencadeamento de incontinência urinária e fecal, distopias genitais e até lacerações perineais importantes (FEITOSA et al., 2017, p.722).

Portanto, ficou evidente que a vivência do processo de parto normal é encarada como um desafio para a mulher. Por isso, as informações relacionadas às vantagens e desvantagens são essenciais para a elucidação das dúvidas referentes ao período de parturição. A decisão da via de parto será mais segura, se a gestante for esclarecida de todo o processo, para assim poder usufruir do seu protagonismo e optar pelo parto normal. Isso a ajudará na construção precoce do vínculo mãe-bebê e trará enormes benefícios para ambos.

\section{CONCLUSÃO}

Os estudos revelaram que o ciclo gravídico puerperal é uma fase marcante na vida da mulher, durante a qual é preciso tomar decisões significativas, que contribuam para uma melhor qualidade de vida do binômio mãe-bebê. Tais decisões têm início na escolha da via de parto, para o qual a mulher deve ter uma orientação adequada, a partir de uma assistência à saúde de qualidade realizada por

\footnotetext{
*A manobra de Kristeller é uma manobra obstétrica executada durante o parto que consiste na aplicação de pressão na parte superior do útero com o objetivo de facilitar a saída do bebê. Fonte: <http://www.corensc.gov.br/wp-content/uploads/2016/06/Parecer-T\%C3\%A9cnico-0012016-CT-Sa\%C3\%BAde-Mulher-Manobra-de-Kristeller.pdf>. Acesso em: 24 mai. 2018.

${ }^{* *}$ A episiotomia é uma incisão cirúrgica no períneo com objetivo de aumentar o canal de parto. Aepisiotomia é uma incisão cirúrgica realizada no períneo da mulher no momento da expulsão - segundo período do parto. Fonte: <http://www.scielo.br/pdf/ean/v10n3/v10n 3a27.pdf>. Acesso em: 24 mai. 2018.

${ }^{* *}$ A episiorrafia é uma suturação realizada após a episiotomia ao qual se utiliza fio categute simples. Fonte: <http://www.scielo.br/pdf/rlae/v23n1/pt_0104-1169-rlae-23-01-00162.pdf>.Acesso em: 24 mai. 2018.
} 
profissionais que acolham essa mulher de uma maneira humanizada e única, possibilitando que os medos e tabus sejam desmistificados e, ao mesmo tempo, encorajando-a e demonstrando a importância do seu protagonismo durante todo o decorrer da parturição.

Além dessas informações, foi possível entender que muitas mulheres optam pela cesariana em detrimento do parto normal, e que este fenômeno pode ser explicado por diversos fatores, como o déficit de informações durante a assistência de pré natal - devido a uma assistência precária ou até mesmo devido à deficiência na comunicação entre a equipe de saúde, a gestante e seus familiares; o receio da dor e do desconhecido; e as crenças, mitos e experiências anteriores de parentes e amigos.

Ademais, compreendeu-se que há necessidade de incentivo, por parte dos profissionais de saúde, sejam eles médicos ou enfermeiros, ao parto normal, devido à contribuição para a melhor qualidade de vida do binômio mãe-bebê, já que suas vantagens incluem a diminuição do risco de taquipnéia transitória e síndrome respiratória; redução do estresse (para o bebê); recuperação mais rápida no pós parto; o protagonismo no parto (para a mulher); diminuição do risco de infecção; e aumento do vínculo do binômio mãe-bebê.

Diante dessas evidências, conclui-se que os profissionais de saúde devem promover a educação em saúde, através da criação de rodas de gestantes, para um maior e melhor acolhimento, incentivando o parto natural e ressaltando os seus benefícios (e suas desvantagens), além do compartilhamento de experiências, com objetivo de promover a autonomia e o empoderamento feminino, prezando sempre a humanização no parto.

\section{REFERÊNCIAS}

CAMPOS, A. S.; ALMEIDA, A. C. C. H de.; SANTOS, R. P dos. Crenças, mitos e tabus de gestantes acerca do parto normal. Rev Enferm. UFSM, 2014 Abr/Mai; 4(2):332-341.

CARNEIRO, L. M. A.; PAIXÃO, G. P do N.; SENA, C. D. de. et al. Parto natural x parto cirúrgico: percepções de mulheres que vivenciaram os dois momentos. R. Enferm. Cent. O. Min. 2015 mai/ago; 5(2):1574-1585.

FEITOSA, R. M. M.; PEREIRA, R. D.; SOUZA, T. J. C. de P. et al. Fatores que influenciam a escolha do tipo de parto na percepção das puérperas. J. res.: fundam. care. online 2017. jul./set. 9(3): 717-726.

FREIRE, H. S. S.; CAMPOS, F. C.; CASTRO, R. C. M. B. et al. Parto normal assistido por enfermeira: experiência e satisfação de puérperas. Rev enferm UFPE online., Recife, 11(6):2357-67, jun., 2017.

GUEDES, G. W.; SOUSA, M. N. A de.; LIMA, T. N. F. de A. et al. Conhecimentos de gestantes quanto aos benefícios do parto normal na consulta pré-natal. Rev enferm UFPE online., Recife, 10(10):3860-7, out., 2016;

MELO, J. K. F.; DAVIM, R. M. B.; SILVA, R. A. R.Vantagens e desvantagens do parto normal e cesariano: opinião de puérperas. J. res.: fundam. care. online 2015. out./dez. 7(4): 3197-3205;

MELO, K. L.; VIEIRA, B. D. G.; ALVES, V. H. et al. O comportamento expresso pela parturiente durante o trabalho de parto: reflexos da assistência do pré-natal. J. res.: fundam. care. online 2014. jul./set. 6(3):1007-1020.

NASCIMENTO, R. R. P do.; ARANTES, S. L.; SOUZA, E. D. C de. et al. Escolha do tipo de parto: fatores relatados por puérperas. Rev Gaúcha Enferm. 2015;36(esp):119-26.

PIMENTA, L. F. et al. A cultura interferindo no desejo sobre o tipo de parto. J. res.: fundam. care. online 2014. jul./set. 6(3):987-997.

PINHEIRO, T. M.; SILVA, S. C da.; BARRETO, C. N. et al. Fatores que influenciam na indicação da via de parto. $\mathbf{R}$. Enferm. Cent. O. Min. 2016 jan/abr; 1(6):2066-2080.

RATTNER, D.; MOURA, E. C. Nascimentos no Brasil: associação do tipo de parto com variáveis temporais e sociodemográficas. Rev. Bras. Saúde Matern. Infant., Recife, 16 (1): 39-47 jan. / mar., 2016. 
RIBEIRO, J. F.; LIMA, M. R.; CUNHA, S. V. et al. Percepção de puérperas sobre a assistência à saúde em um centro de parto normal. Rev Enferm UFSM 2015 Jul./Set.;5(3):521-530.

SANTOS, C. L dos.; BORTOLI, C. de F. C de.; PRATES, L. A. et al. Preparo e percepções de gestantes sobre as vias de parto. Rev Enferm UFSM 2016 Abr./Jun.;6(2): 186-197.

SCARTON, J.; PRATES, L. A.; WILHELM, L. A. et al. "No final compensa ver o rostinho dele": vivências de mulheresprimíparas no parto normal. Rev Gaúcha Enferm. 2015; 36(esp):143-51.

SILVA, S. P. C.; PRATES, R. C. G.; CAMPELO, B. Q. A. Parto normal ou cesariana? Fatores que influenciam na escolha da gestante. Rev Enferm UFSM 2014 Jan/Mar;4(1):1-9.

SOARES, C. B.; HOGA, L. A. K.; PEDUZZI, M. et al. Revisão integrativa: conceitos e métodos utilizados na enfermagem. Rev Esc Enferm USP. 2014; 48(2):335-45.

VARGAS, P. B.; VIEIRA, B. D. G.; ALVES, V. H. et al. A assistência humanizada no trabalho de parto: percepção das adolescentes. J. res.: fundam. care. online 2014. jul./set. 6(3):1021-1035.

VELHO, M. B.; SANTOS, E. K. Ados.; COLLAÇO, V. S. Parto normal e cesárea: representações sociais de mulheres que os vivenciaram. Rev Bras Enferm. 2014 mar-abr; 67(2): 282-9. 